\title{
SENSITIVITY ANALYSIS ON THE EFFECT OF MEASUREMENT NOISE AND SAMPLING FREQUENCY ON THE CALCULATION OF THE TEMPORAL LIGHT ARTEFACTS
}

\author{
Thorseth, Anders; Linden, J.; Bouroussis, C. A.
}

Published in:

Proceedings of the Conference CIE 2021

Link to article, DOI:

10.25039/x48.2021.OP28

Publication date:

2022

Document Version

Publisher's PDF, also known as Version of record

Link back to DTU Orbit

Citation (APA):

Thorseth, A., Linden, J., \& Bouroussis, C. A. (2022). SENSITIVITY ANALYSIS ON THE EFFECT OF MEASUREMENT NOISE AND SAMPLING FREQUENCY ON THE CALCULATION OF THE TEMPORAL LIGHT ARTEFACTS. In Proceedings of the Conference CIE 2021 (pp. 245-254). CIE - International Commission on Illumination. https://doi.org/10.25039/x48.2021.OP28

\section{General rights}

Copyright and moral rights for the publications made accessible in the public portal are retained by the authors and/or other copyright owners and it is a condition of accessing publications that users recognise and abide by the legal requirements associated with these rights.

- Users may download and print one copy of any publication from the public portal for the purpose of private study or research.

- You may not further distribute the material or use it for any profit-making activity or commercial gain

- You may freely distribute the URL identifying the publication in the public portal 


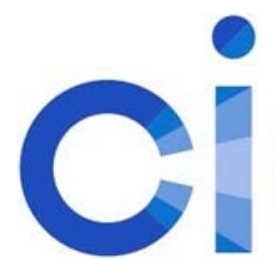

International Commission on Illumination

Commission Internationale de l'Eclairage

Internationale Beleuchtungskommission

OP28

\section{SENSITIVITY ANALYSIS ON THE EFFECT OF MEASUREMENT NOISE AND SAMPLING FREQUENCY ON THE CALCULATION OF THE TEMPORAL LIGHT ARTEFACTS}

Thorseth, A., Lindén, J., Bouroussis, C.A.

DOI 10.25039/x48.2021.OP28

Pages 245-254 from

CIE x048:2021

Proceedings of the Conference

CIE 2021

(DOI 10.25039/x48.2021)

The paper was presented at the Conference CIE 2021, hosted by NC Malaysia online, September 27-29, 2021. It has not been peer-reviewed by the CIE.

(C) CIE 2021

All rights reserved. Unless otherwise specified, no part of this publication may be reproduced or utilized in any form or by any means, electronic or mechanical, including photocopying and microfilm, without permission in writing from CIE Central Bureau at the address below.

CIE Central Bureau

Babenbergerstrasse 9

A-1010 Vienna

Austria

Tel.: +4317143187

e-mail: ciecb@cie.co.at

www.cie.co.at 


\title{
SENSITIVITY ANALYSIS ON THE EFFECT OF MEASUREMENT NOISE AND SAMPLING FREQUENCY ON THE CALCULATION OF THE TEMPORAL LIGHT ARTEFACTS
}

\author{
Thorseth, A. ${ }^{1}$, Lindén, J. ${ }^{2}$, Bouroussis, C.A. ${ }^{3}$ \\ 1 Technical University of Denmark, Roskilde, DENMARK, ${ }^{2}$ Division of Ergonomics and Aerosol \\ Technology, Design Sciences, Lund University, Lund, SWEDEN, ${ }^{3}$ Lighting Laboratory, National \\ Technical University of Athens, GREECE \\ andt@fotonik.dtu.dk
}

DOI $10.25039 / \times 48.2021 .0 P 28$

\begin{abstract}
Temporal light modulation (TLM) and the resulting temporal light artefacts (TLA) can cause problems with health and wellbeing for users of lighting products. Therefore, TLM has to be measured accurately and repeatably. This study investigates important factors influencing the measurement uncertainty of TLM measurements. The study shows how measurement uncertainty on central TLM parameters can have a significant effect on the calculation of TLA. Specifically, we show a linear relationship between DC offset and the expected error. Further we show severe effects of random noise on PstLM on certain waveforms. And lastly, we show a curious effect related to SVM of pulse width modulated signals and the measurement sampling frequency.
\end{abstract}

Keywords: Photometry, Temporal light modulation, TLM, Temporal light artefacts, TLA, Flicker, Measurement uncertainty, Propagation of uncertainty.

\section{Introduction}

The human visual system is well adapted to notice sudden changes in the visual environment, for instance for fast detection of predators or other dangers. Periodic modulation, however, has only recently been introduce to our environment by electric lighting, causing problems of health and comfort (Veitch et al., 2021). Here the temporal light modulation of light (TLM) is on a frequency scale resembling that of sound, and for LED lighting in some cases even higher. This short time scale is somewhat new to the lighting community, so a lot of concepts have had to be introduced in recent years, increasingly with the emergence of LED lighting, such as frequency domain measurements, frequency filtering etc.

TLM is generally caused by LED sources powered by modulated power supplies which introduce modulation of the light output. This leads to specific visual artefacts when seen by a human observer. TLM are typically more severe in low quality- and in most cases, low priced LED lighting products. It would be unfortunate if the benefits from the increase in energy efficiency from adoption of LED lighting technology would be offset, by the negative consequences of temporally modulated artefacts (TLA). This issue has been of high research interest in recent years and resulted in specific acts against it.

The EU market regulation 2019/2020, which will replace Regulations No 244/2009, No 245/2009 and No 1194/2012 and will be in force starting on September 2021 (European Commission, 2020), includes restrictions against TLA in lighting products. More specifically, EU regulation recognises the 'stroboscopic effect' as a change in motion perception induced by a light stimulus, the luminance or spectral distribution of which fluctuates with time, for a static observer in a non-static environment. The metric for the stroboscopic effect used in this regulation is the 'SVM' (stroboscopic visibility measure) described in detail by the IEC (IEC TC 34,2018 ). The same regulation recognises 'flicker' as the perception of visual unsteadiness induced by a light stimulus, the luminance or spectral distribution of which fluctuates with time, for a static observer in a static environment. The metric for flicker used in this regulation is the parameter 'PstLM', also described in detail by the IEC (IEC TC 34, 2017). According to this EU regulation, TLM for LED and OLED products should result in PstLM less or equal than 1.0 at full-load, while the stroboscopic effect for the same products should be less or equal to 0.9 at full-load, with that limit expected to change to 0.4 in 2024. Regarding the verification tolerances 
during market surveillance campaigns, values of PstLM and SVM shall not exceed the declared value by more than $10 \%$.

Both of these TLA metrics are well defined and discussed in the CIE Technical Note 006:2016 "Visual Aspects of Time-Modulated Lighting Systems - Definitions and Measurement Models" (CIE, 2016) which was a product of the dedicated Technical Committee CIE TC 1-83 Visual Aspects of Time-Modulated Lighting Systems. Another CIE Technical Committee dedicated to this subject (TC 2-89) is actively working on the preparation of a Technical Report and an International Standard on the "Measurement of Temporal Light Modulation of Light Sources and Lighting Systems", having already published a Technical Note CIE TN 012:2021(CIE, 2021). An EU funded research project EMPIR MetTLM 20NRM01 on the metrology of temporal lighting modulation will be running from 2021 to 2024, dealing with specific metrological aspects of this complicated issue (Dekker and et al., 2021).

In practice, the measurement of TLM and TLA is expected to take place in both laboratory conditions and in the field. Current measurement methods incorporate a variety of instruments, e.g., handheld, benchtop, camera based, etc. Each instrument may use different sampling frequencies (fixed or signal dependent). In addition, measurements may be affected by noise and for field measurements, other light sources that cannot be properly shielded or switched off. Since the measures of TLM and TLA are time domain and frequency domain related, a dedicated signal processing algorithm should be applied. It is clear that the final reported value of PstLM and SVM should be accompanied with statements of uncertainty, sourced from measurements as well as from mathematical calculations on the measured signals. Propagation of uncertainty in Fourier transforms considering the GUM(Joint Committee for Guides in Metrology (JCGM), 2008) has been studied (Betta et al., 2000; Eichstädt and Wilkens, 2016), but not to our knowledge for TLM. In this study we have opted for a Monte Carlo style approach, feeding the algorithms with a large number of input parameters and investigating the output. This deals with the fact that the data set of a TLM measurement can potentially consist of millions of individual data points, but due to the periodic nature of the source the individual values will be highly correlated.

This study is an investigation of the propagation of uncertainty through the calculation of the TLA metrics; PstLM and SVM, when affected by offset, random measurement noise, as well as the sampling frequency during measurements. The results of this study show that there are certain aspects in the calculation of the TLA metrics that can be significantly affected by measurement uncertainties and the method of measurement.

\section{Methods}

This study includes an investigation of the calculation of PstLM and SVM (2.1) on a predefined set of computationally generated light waveforms (2.2), subjected to, variation in DC offset (2.3), random noise (2.4) and variations in the sampling frequency (2.5). The offset is applied as a straightforward parameter variation while the application of random noise is investigated through Monte Carlo simulation. In this work we have not investigated the correlation between the individual effects. It could for instance be expected that electrical offset (typically decreases TLA) and electrical noise (typically increases TLA) would be correlated. The study of sampling frequency is conducted by varying the frequency of a simulated PWM waveform, while applying certain different sampling frequencies.

\subsection{TLA calculations}

The TLA calculations in this study where made using the MATLAB based tool "Stroboscopic effect visibility measure toolbox" (Beeckman and Sekulovski, 2018) for the SVM, based on the IEC standard (IEC TC 34, 2018), and the "Light flicker assessment toolbox" (Beeckman, 2017) for PstLM, based on the IEC standard (IEC TC 34, 2017).

Before the waveforms are subjected to TLA calculations, all negative values caused by uncertainty effects are set to zero. This is done to accommodate the calculation of TLA. How this specific method of truncation may influence the results, has not been investigated. 


\subsection{Test waveforms}

In this study we have chosen to use a set of predefined waveforms representing typical behaviour of TLM measured in the field: Waveform $W_{1}$ represents an ideally stable and TLMfree light source, the subsequent waveforms are: A rectified sine wave $\left(W_{2}\right)$, an offset sine wave $\left(\mathrm{W}_{3}\right)$, pulse width modulation (PWM) signals with various offsets and frequencies $\left(\mathrm{W}_{5}\right.$ to $\left.\mathrm{W}_{12}\right)$ as well as various sawtooth waves $\left(\mathrm{W}_{13}\right.$ to $\left.\mathrm{W}_{16}\right)$ and other types defined to stress-test the algorithms and methods using more uncommon waveforms. The waveforms were generated using mathematical functions and have been normalized to the average value and are clear from noise or other artefacts. Figure 1 illustrates a few periods of each waveform. The total duration of each generated waveform is $180 \mathrm{sec}$.
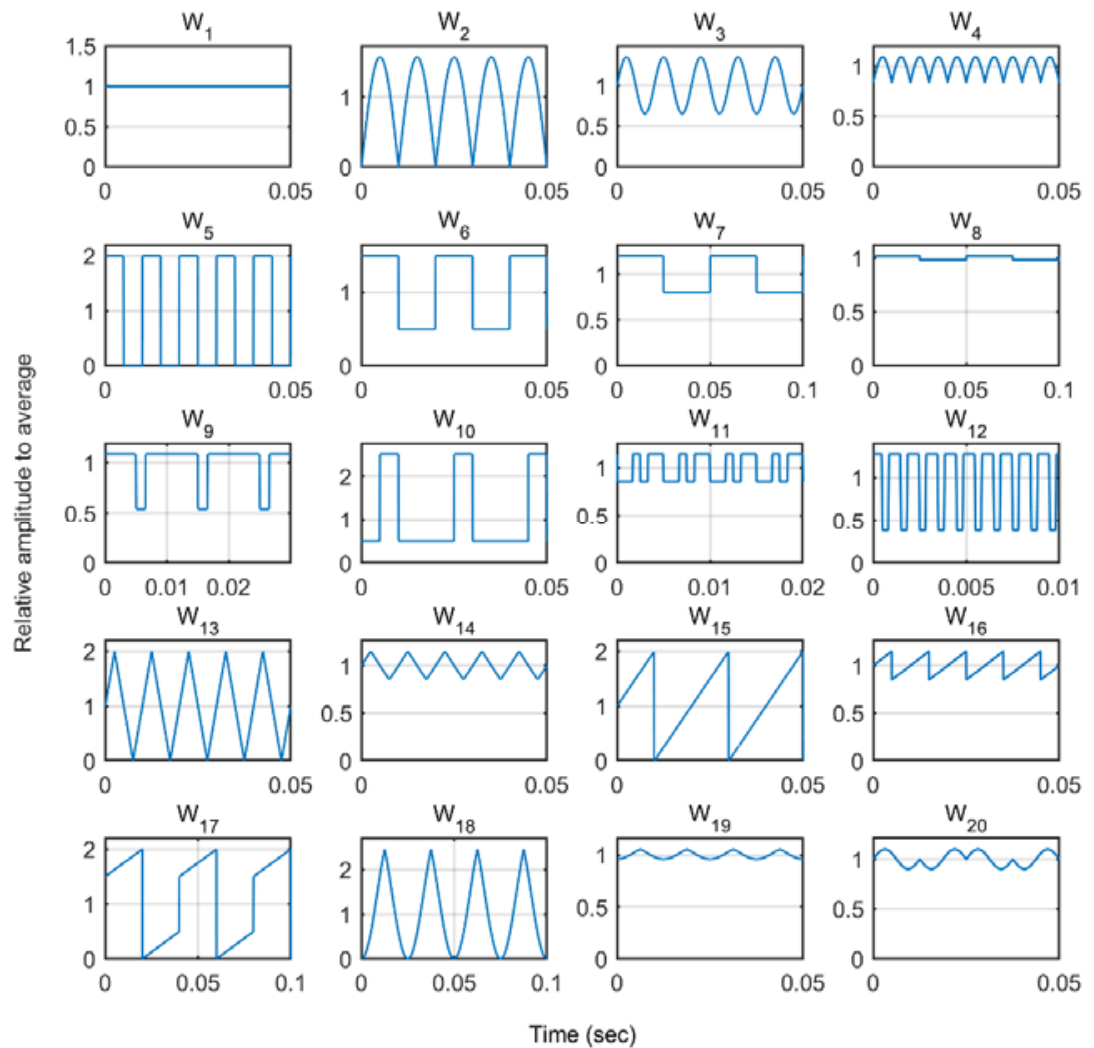

Figure 1 - The computationally generated test waveforms used in this study, numbered $\mathrm{W}_{\mathrm{n}}$, $n=1 \ldots 20$.

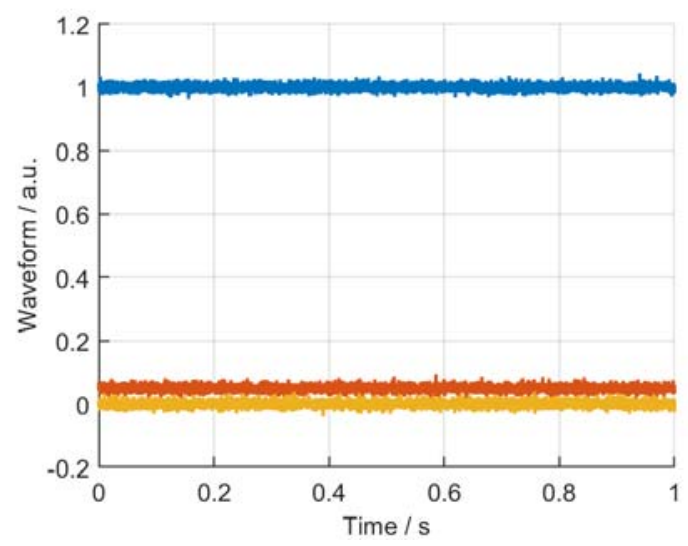

a)

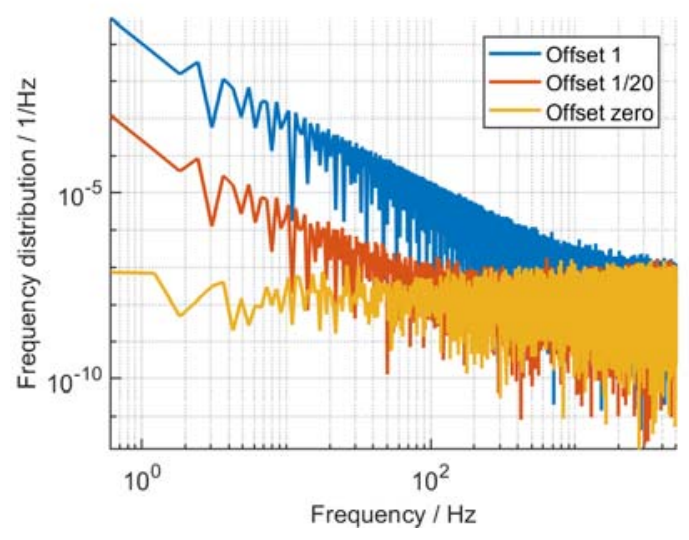

b)

Figure 2 - Effect on the fast Fourier transform on a DC waveform with a small amount of noise (a), when applying an offset, of zero, 1/20 and unity, the FFTs are illustrated (b). 


\subsection{Offset}

When doing measurements, it is very common to have an offset (or DC component) on the measured data. Many electrical circuits produce this unintentionally, and this offset value can be affected by changes in sensor temperature or other environmental effects. External straylight from steady sources such as daylight will also produce an offset. In addition, non-perfect zeroing of the light sensor may introduce an offset or a clipping of the waveform on its lower part. In some cases, the offset can be corrected, by doing a measurement with the device under test turned off. However, this correction will carry its own uncertainty. The effect on the frequency spectrum introduced by an offset is illustrated in Figure 1. Here it can be seen that the fast Fourier transform (FFT) of a waveform with an added offset changes the frequency spectrum of a mildly noisy signal ( $1 \%$ noise) by adding more low frequency components. This is due to the DC component of the waveform acting as a single square wave, with all the harmonic content from such a signal. In practice, DC offsets have random elements, however, in this study, the DC offsets are considered as flat (stable) signals of a certain amplitude. In 3.1 we present the results of varying the offset on the test waveforms of Figure 1 . All waveforms, except $\mathrm{W}_{1}$, subjected to an offset show a sensitivity between $-1 \% / \%$ and $-2.5 \% / \%$, in the TLA calculations.

\subsection{Random noise}

Completely uncorrelated random noise (white noise) often seen in electrical circuits as well as other forms of random noise, changes the waveform frequency distribution by raising the general level of all frequencies. Amplifiers with high gain and fast reaction time can increase the noise level by disproportionally amplifying small changes in the original signal. A common way to reduce high frequency noise is to apply low pass filters.

The noise used in this study is computationally generated normal/Gaussian distributed values added to a given waveform with a standard deviation set to a percentage of the maximum value of the waveform. It's important to consider that normal distributions are not bounded, so extreme values can occur, even though with low probability. In this study no filtering has been applied to the waveforms after application of the noise.

It is important to consider any filtering in relation to noise since the filter will change the distribution of noise in the measurement system. Using high- and low pass filters will bound the noise in frequency but not eliminate it. The results presented in 3.2 show severe effects for PstLM on some of the test waveforms, while the effect on SVM is very limited.

\subsection{Sampling frequency}

An important aspect of TLA analysis of TLM, such as calculating the SVM or PstLM, is the sampling frequency, Fs, used during measurements. According to CIE TN 012(CIE, 2021), the minimum recommended $F s$ is $20 \mathrm{kHz}$. Since the SVM is defined only for frequencies below $2000 \mathrm{~Hz}$, this minimum recommendation should be well enough for sufficient analysis of the waveform, according to the Nyquist criteria. The MATLAB function (Beeckman and Sekulovski, 2018) accepts however a minimum sampling frequency of $4 \mathrm{kHz}$. In 3.3 it is shown that the choice of sampling frequency can have a severe effect on the obtained results.

\section{Results}

This section describes the result of SVM and PstLM calculated on the test waveforms (2.2) for varying offset (3.1), noise level (3.2) and sampling frequency (3.3).

\subsection{Offset}

A DC offset was added to all waveforms varying from -5 to $+5 \%$ of the maximum of each signal in several steps. For the waveforms with $100 \%$ modulation depth, the resulting negative values were omitted during the TLA calculations. In all cases, the DC offset was free of noise or other artefacts. The effect on TLA values when adding an offset to the test waveforms can be seen in Figure 3. Unintentional offsets are often positive, however negative offsets are also possible, for instance when applying an erroneous correction. It is seen that for small offsets the change is very nearly linear. The general tendency is a negative slope, described as follows: $E=k \cdot x$, where $E$ is the deviation (in \%) of the calculated PstLM or SVM value compared to the reference 
value, $x$ is the DC offset on the input signal as \% of the maximum value, and $k$ is the coefficient (slope). It should be noted that both TLA metrics of a given waveform are affected in the same way by the added offset. Therefore, the vertical axis of Figure 3 refers to both TLA values.

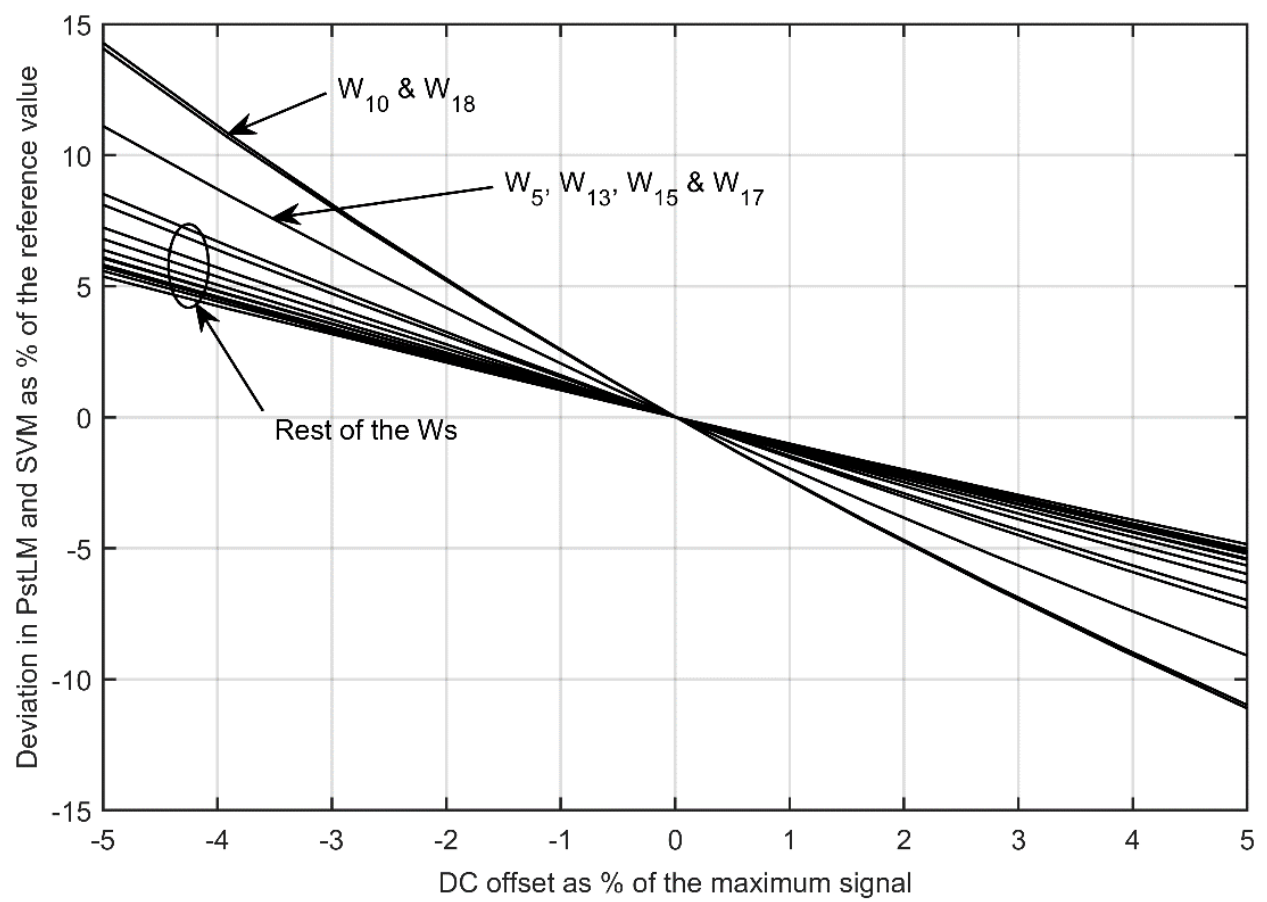

Figure 3 - Deviation in PstLM and SVM values when a DC offset is applied to the test waveforms.

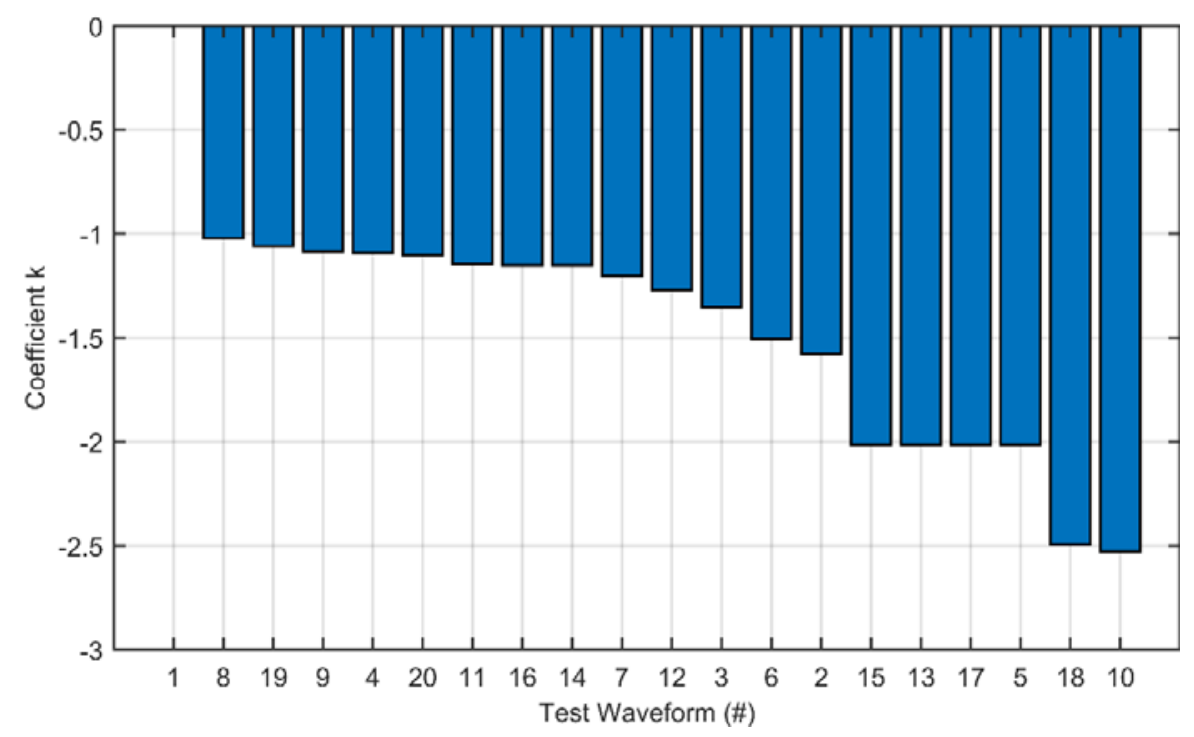

Figure 4 - Slope of the changes in PstLM and SVM for changing the DC offset seen in Figure 3. The results are sorted according to effect magnitude.

It is seen in Figure 3 and Figure 4 that the slope is varying between -1 and -2.5 , meaning that an $1 \%$ positive offset will be translated to between $1 \%$ and $2.5 \%$ decrease in TLA value. The majority of the test waveforms show a reduction of TLA values from 1 to $\sim 1.5 \% / \%, W_{5}, W_{13}, W_{15}$ and $W_{17}$ a reduction of $\sim 2 \% / \%$, and $W_{10}$ and $W_{18}$ a reduction of $2.5 \% / \%$. 


\subsection{Random noise}

Random noise is a common occurrence both in electrical circuitry and from other sources for instance in field measurements. The origin of PstLM, related to disturbances in the power grid, shows itself when adding random noise to the test waveforms and calculating PstLM, due to the fact that random noise contains a small number of values far from the mean value, these values will be interpreted as disturbances and cause an increase in PstLM. Figure 5 shows the result of a Monte Carlo simulation with 100 random signals added to each test waveform for five cases of random noise levels varying in standard deviation from $0 \%$ to $1 \%$ of the maximum of the test waveform.

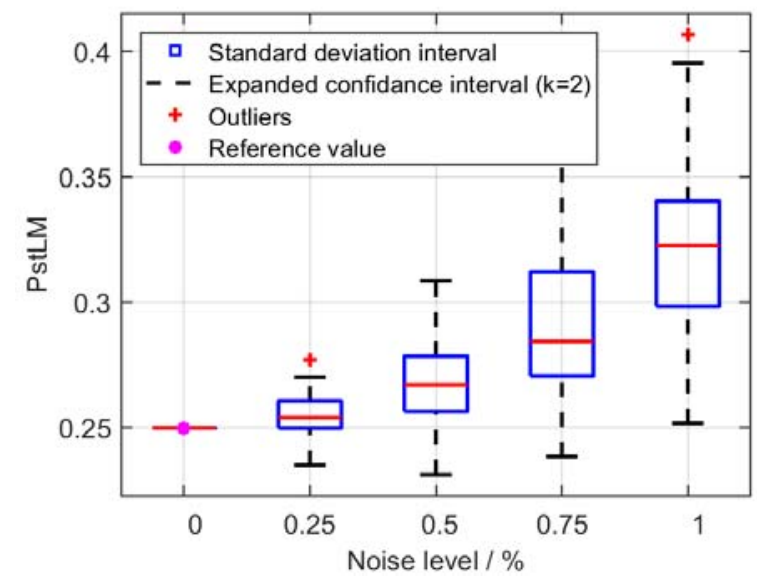

a)

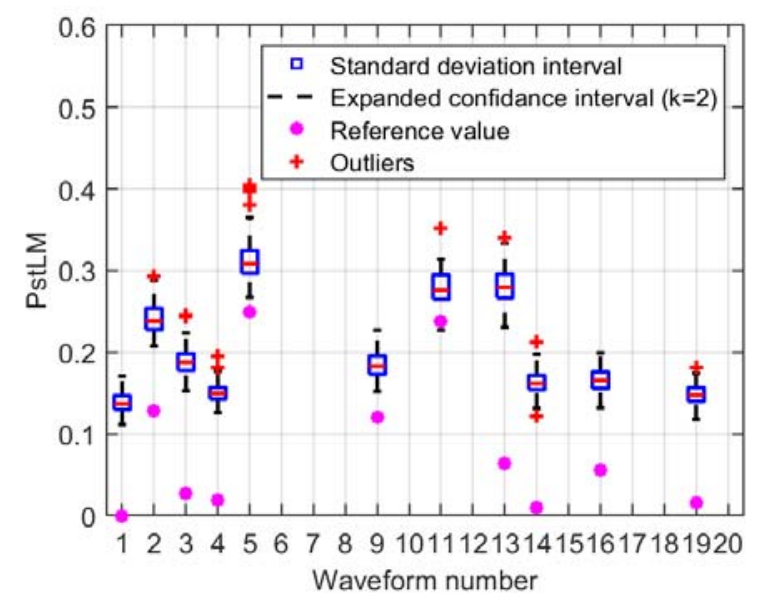

b)

Figure 5 - Results of the Monte Carlo simulation, adding random noise. The spread of PstLM values for a single representative waveform $\left(W_{5}\right)$ for 100 different noise signals added per noise level (a), and for a 1\% noise level shown for the waveforms with PstLM values below 1.0 (b).

As seen in Figure 5, the calculated PstLM values are generally shifted (by $\Delta_{P s t L M}$ ) upward from the reference value with increasing noise level (see Figure 6a). Since the mean value and the reference value don't align, this shift cannot be corrected by repeating the measurement with a similar noise level and calculation of the mean. This method can only account for the variation in results, seen in Figure 6b. The standard deviation in the absolute value of PstLM from random noise is limited to values below the line with the slope $0.025 / \%$. For high values of PstLM, introduction of noise can lower the value (especially seen for $W_{18}$ ).

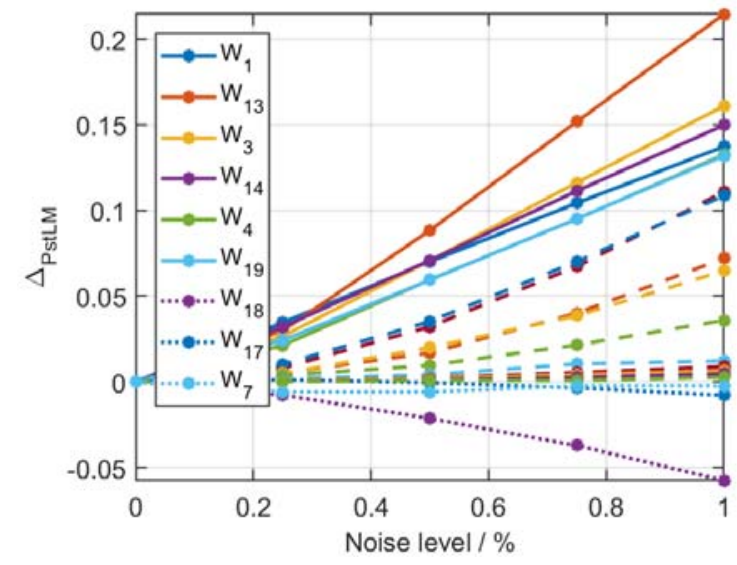

a)

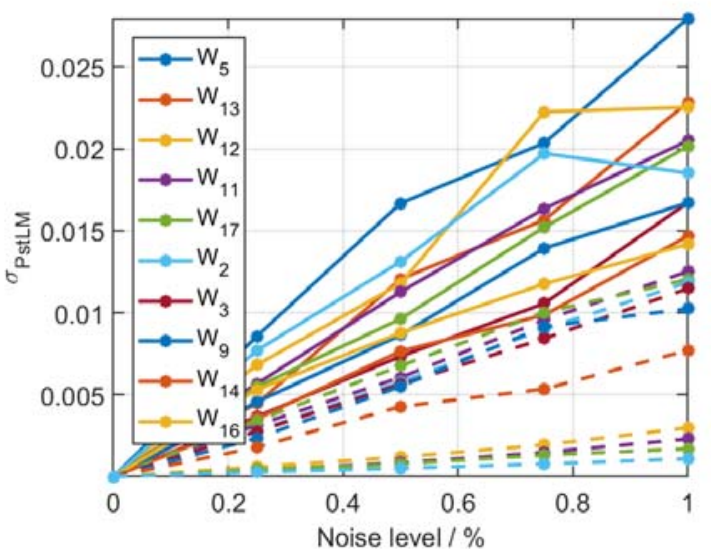

b)

Figure 6 - The shift of PstLM values $\Delta_{P s t L M}(\mathrm{a})$, negative shifts are marked with dotted lines. The standard deviation of PstLM values $\sigma_{P s t L M}(\mathrm{~b})$. Legends are sorted to denote the waveforms with the largest deviation, waveforms with low change is marked with dashed lines. 


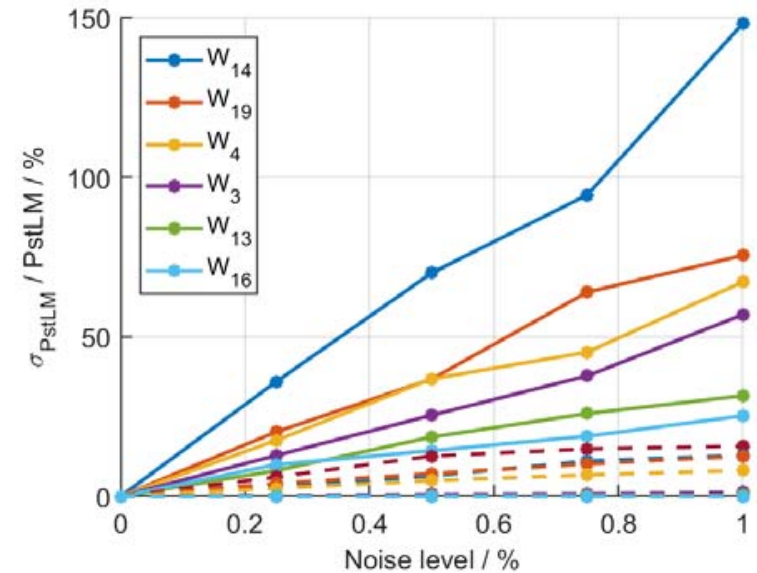

a)

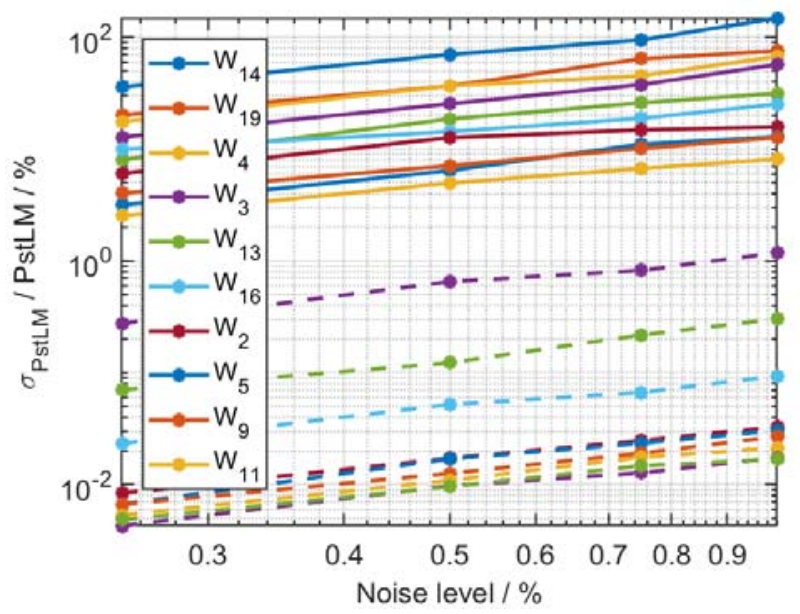

b)

Figure 7 - The relative standard deviation of PstLM values $\sigma_{P s t L M}$ plotted in a linear plot (a) and a double logarithmic plot (b). Legends are sorted to denote the waveforms with the largest deviation, waveforms with low relative change is marked with dashed lines.

The spread of PstLM results from random noise presents straight lines in a double logarithmic plot (Figure 7b), with approximately the same slope. This means that the relationship can be approximated by formular $y=a x^{k}$ where $y$ is the standard deviation, $x$ is the noise level and $k$ is approximately constant for all shown experiments, with $k=1.0 \pm 0.3$. This means that a good approximation of the uncertainty associated with noise will be described by a linear relationship with the noise level. In Figure 7 it is seen that the standard deviation from a $1 \%$ random signal added on the waveform creates significant uncertainty in the range near PstLM $=1.0$, which is the EU limit for PstLM (European Commission, 2020).

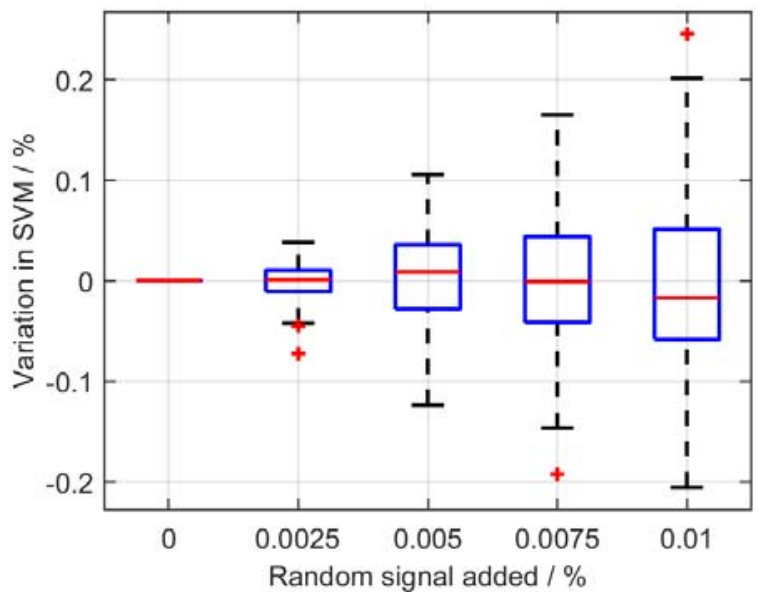

a)

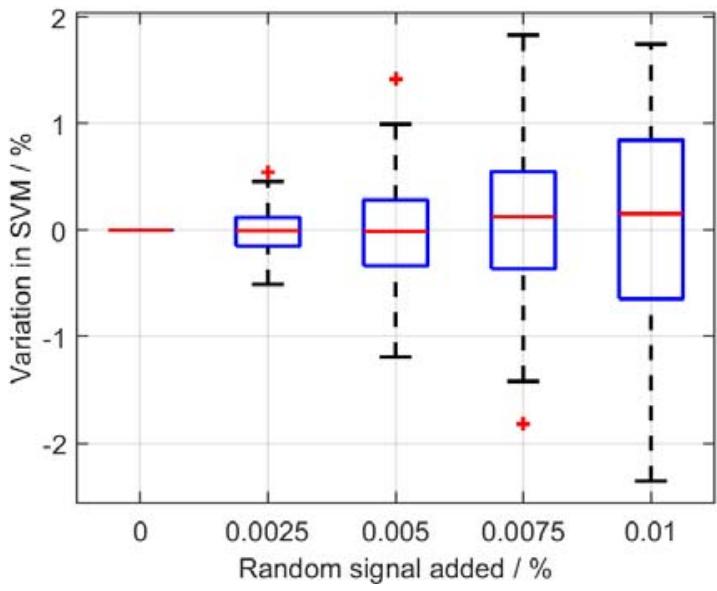

b)

Figure 8 - A typical variation in SVM due to random noise (Waveform $\mathbf{W}_{4}$ shown) The worst case of relative change is observed is for $W_{8}(b)$.

As SVM is not designed as a measure of noise, the effect of introduced noise is limited. It is seen from Figure 8 that the standard deviation in SVM from random noise is approximately proportional to $1 / 10$ of the random noise added to the signal. Here it can be seen that the average value is near to the reference value which means it can be beneficial to repeat and average the measurement for higher accuracy. However, it should be noted that this effect is quite small compared to the other effects investigated in this study. 


\subsection{Sampling frequency}

The effect of sampling frequency is investigated for the special case of artificial square waveforms (100\% modulation depth and $65 \%$ duty cycle). The SVM is calculated for a range of PWM frequencies from 10 to $2000 \mathrm{~Hz}$, with steps of $0.1 \mathrm{~Hz}$, i.e. 19901 different frequencies. The duration is 2 seconds, and no additional noise is added to the waveforms. The SVM for the entire range is calculated for 5 different sampling frequencies, Fs (see Figure 9).

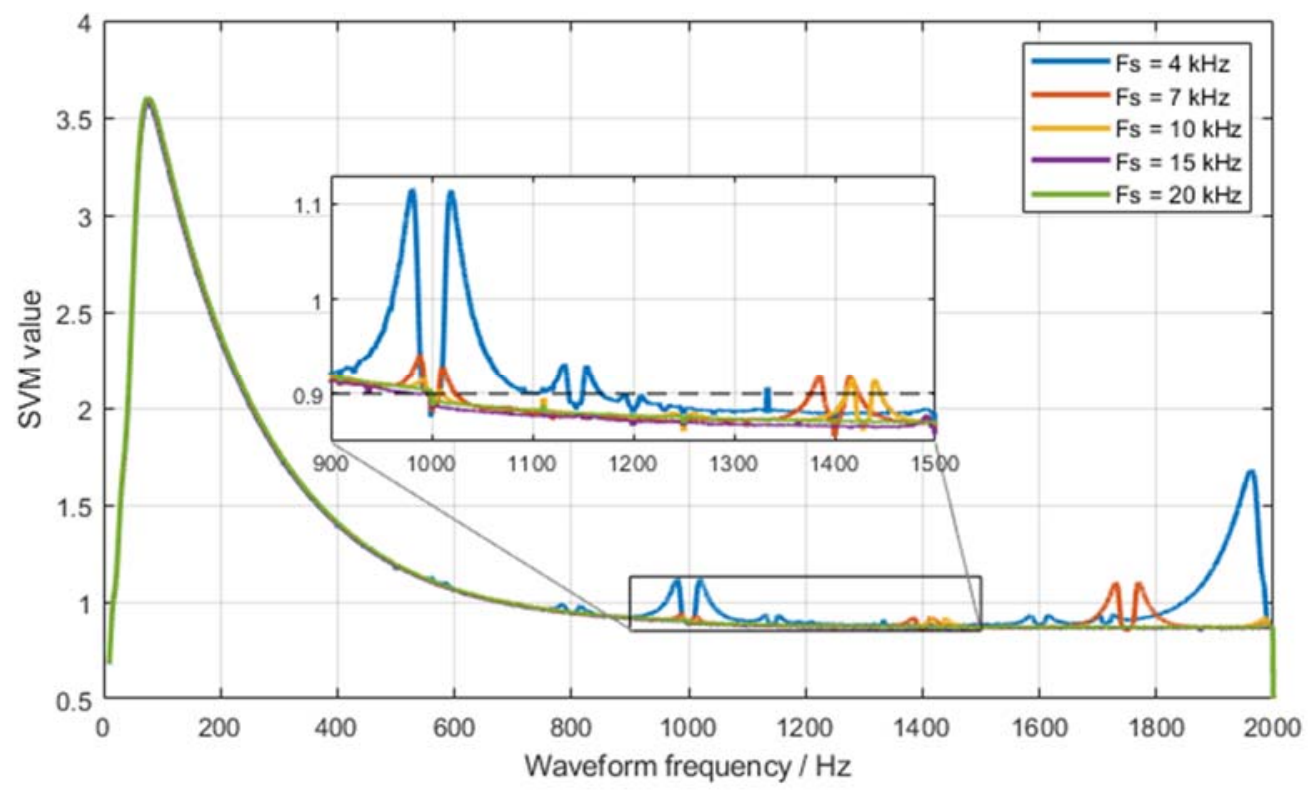

Figure 9 - SVM values as a function of PWM waveform frequency, calculated at different sampling frequencies $\mathrm{Fs}$, for square waves (100\% modulation depth $65 \%$ duty cycle). Subfigure shows a zoomed area corresponding to the rectangle. The dashed black line indicate the EU regulation limit of SVM $=\mathbf{0 . 9}$.

From Figure 9 it can be seen that the lower the Fs, the more prominent is the appearance of some symmetrical artefacts, here called wing-artefacts. As can be seen in the subfigure, showing a zoomed in area between $900 \mathrm{~Hz}$ and $1500 \mathrm{~Hz}$, these wing-artefacts are present at all sampling frequencies, albeit to lesser extent with increasing sampling frequency, also in the highest Fs of $20 \mathrm{kHz}$. The origin of these wing-artefact is at the time of writing unknown, but it is suspected they originate from the calculation algorithm of the SVM value. As can be seen in the case of $\mathrm{Fs}=4 \mathrm{kHz}$, the SVM values around waveform frequency $1000 \mathrm{~Hz}$ fluctuates between 0.9 and 1.1. For lower duty cycles this fluctuation is much more prominent. In this example, the duty cycle is selected to $65 \%$, since this value results in fluctuations around the limit of SVM $=0.9$ (indicated by the dashed line in Figure 9), which is included in the coming EU regulation.

Although the above result is obtained using artificial waveforms, these wing-artefacts will still be present in low noise real-world measurements. The impact of small differences in frequencies on the precision of SVM considering real-world waveforms are under investigation, but it is expected the effect is significant.

\section{Conclusions and outlook}

\subsection{Conclusions}

Measurement of TLM and calculation of TLA are sensitive to measurement uncertainties. As TLA metrics, PstLM and SVM, are becoming critical market parameters, these uncertainties should be investigated and documented. This study contributes to the understanding of the effect of offset, random noise and sampling frequency on the calculated values of PstLM and SVM, which in some cases can be significant. These issues are likely to be of high importance in relation to the regulations stated in the updated EU eco design directives, entering into force 
in September 2021(European Commission, 2020). The main results of the study can be summarized as follows:

- Offset of waveforms (caused by electrical issues in the measurement instrument, environmental stray light, etc.) is shown to produce a relative error in PstLM and SVM between -1 and -2.5 times the relative offset.

- We find that random noise can have a severe effect on PstLM, depending on the waveform. This effect can possibly cause both a shift and a spread in PSTLM values from a noisy signal. On the other hand, the effect of noise on SVM is low or negligible.

- We demonstrate apparent calculation artefacts of unknown origin in calculations of SVM depending on the frequency of PWM signals and sampling frequency, especially pronounced for low sampling frequency.

The result on offset suggests that a clear procedure for zeroing the equipment and elimination of stray light should be available.

Especially for PstLM and noise there seems to be a non-trivial relation between the magnitude of the uncertainty and the magnitude of the true value. For low values of PstLM the requirement of a maximum $10 \%$ deviation from the declared value is quite restrictive, as noise seems to cause a high relative uncertainty for small values measured. Generally, the uncertainty seem to be highly related to the shape of the waveform.

The results regarding sampling frequency suggests that, in order to have comparable values, sampling frequency should perhaps be required to be set to a single standardised value and not be selectable from above a minimum. The recommended minimum of the sampling frequency in CIE TN 012(CIE, 2021) is $20 \mathrm{kHz}$. However, the available MATLAB function for calculating the SVM (Beeckman and Sekulovski, 2018) allows sampling frequency down to $4 \mathrm{kHz}$. It is suggested that this limit is increased to at least $20 \mathrm{kHz}$.

\subsection{Outlook}

In this paper we have dealt with part of the issues likely to affect TLM measurements. However, the subject is not yet sufficiently covered to form a complete uncertainty budget. Other contributions to a combined uncertainty would be:

- non-linearity (in time domain, and in frequency domain)

- range issues (truncation/saturation/clipping)

- insufficient bit-resolution

- frequency uncertainty

- correlation between all the various uncertainty contributions

Besides these contributions other remaining questions include:

- Since the same measurement uncertainty effect (offset, noise etc.) are clearly affecting different waveforms differently, can TLM waveforms be categorized in terms of "measurement difficulty", such that laboratories can prepare accordingly, with only preliminary knowledge of the waveform?

- Is it possible to use a point by point comparison of two waveforms (Thorseth et al., 2019) to infer what uncertainty contributions might be in play in one or the other?

- The test waveforms presented and used in this study are very preliminary. How would an optimal set of test waveform be defined for use as reference for further research and validation purposes? 


\section{Acknowledgements}

This project (20NRM01 MetTLM) has received funding from the EMPIR programme co-financed by the Participating States and from the European Union's Horizon 2020 research and innovation programme.

\section{References}

BEECKMAN, P., 2017. Light flicker assessment toolbox version 1.2.0.0 [WWW Document]. MATLAB Cent. File EXch.

URL https://www.mathworks.com/matlabcentral/fileexchange/63445-light-flickerassessment-toolbox

BEECKMAN, P., SEKULOVSKI, D., 2018. Stroboscopic effect visibility measure toolbox, version version 1.2.0.0 [WWW Document]. MATLAB Cent. File Exch.

URL https://se.mathworks.com/matlabcentral/fileexchange/59242-stroboscopic-effectvisibility-measure-toolbox

BetTA, G., LIGUORI, C., PIETROSANTO, A., 2000. Propagation of uncertainty in a discrete Fourier transform algorithm. Measurement 27, 231-239. doi:10.1016/S0263-2241(99)00068-8

CIE, 2021. CIE TN012:2021 Guidance on the Measurement of Temporal Light Modulation of Light Sources and Lighting Systems. CIE (Commission International d'Eclairage), Vienna. doi:10.25039/TN.012.2021

CIE, 2016. CIE TN 006:2016 Visual Aspects of Time-Modulated Lighting Systems - Definitions and Measurement Models. Vienna, Austria.

DEKKER, P., ET AL., 2021. Publishable Summary for 20NRM01 MetTLM Metrology for temporal light modulation [WWW Document]. URL http://mettlm.eu

EICHSTÄDT, S., WILKENS, V., 2016. GUM2DFT-a software tool for uncertainty evaluation of transient signals in the frequency domain. Meas. Sci. Technol. 27, 055001. doi:10.1088/0957-0233/27/5/055001

EUROPEAN COMMISSION, 2020. laying down ecodesign requirements for light sources and separate control gears pursuant to Directive 2009/125/EC of the European Parliament and of the Council and repealing Commission Regulations (EC) No 244/2009, (EC) No 245/2009 and (EU) No 1194/2012.

IEC TC 34, 2018. IEC TR 63158:2018 Equipment for general lighting purposes - Objective test method for stroboscopic effects of lighting equipment.

IEC TC 34, 2017. IEC TR 61547-1:2017 Equipment for general lighting purposes - EMC immunity requirements - Part 1: An objective light flickermeter and voltage fluctuation immunity test method.

JOINT COMMITTEE FOR GUIDES IN METROLOGY (JCGM), 2008. JCGM 100:2008 Evaluation of measurement data - Guide to the expression of uncertainty in measurement.

THORSETH, A., LINDÉN, J., BERGEN, A.S.J., 2019. MEASURING AND COMPARING WAVEFORMS OF TEMPORAL LIGHT MODULATION, in: PROCEEDINGS OF the 29th Quadrennial Session of the CIE. International Commission on Illumination, CIE, pp. 7-16. doi:10.25039/x46.2019.OP02

VEITCH, J.A., MARTINSONS, C., COYNE, S., DAM-HANSEN, C., 2021. Correspondence: On the state of knowledge concerning the effects of temporal light modulation. Light. Res. Technol. 53, 89-92. doi:10.1177/1477153520959182 